\section{Inequities in access to mammographic screening in Brazil}

\author{
Inequidades no acesso ao rastreamento por \\ mamografia no Brasil
}

\author{
Mário Cirio Nogueira 1 \\ Vivian Assis Fayer 1 \\ Camila Soares Lima Corrêa 1 \\ Maximiliano Ribeiro Guerra 1 \\ Bianca De Stavola 2 \\ Isabel dos-Santos-Silva 2 \\ Maria Teresa Bustamante-Teixeira 1 \\ Gulnar Azevedo e Silva 3
}

doi: 10.1590/0102-311X00099817

\begin{abstract}
Our objectives with this study were to describe the spatial distribution of mammographic screening coverage across small geographical areas (microregions) in Brazil, and to analyze whether the observed differences were associated with spatial inequities in socioeconomic conditions, provision of health care, and healthcare services utilization. We performed an area-based ecological study on mammographic screening coverage in the period of 2010-2011 regarding socioeconomic and healthcare variables. The units of analysis were the 438 health micro-regions in Brazil. Spatial regression models were used to study these relationships. There was marked variability in mammographic coverage across micro-regions (median $=21.6 \%$; interquartile range: $8.1 \%$ 37.9\%). Multivariable analyses identified high household income inequality, low number of radiologists/100,000 inhabitants, low number of mammography machines/10,000 inhabitants, and low number of mammograms performed by each machine as independent correlates of poor mammographic coverage at the micro-region level. There was evidence of strong spatial dependence of these associations, with changes in one micro-region affecting neighboring micro-regions, and also of geographical heterogeneities. There were substantial inequities in access to mammographic screening across micro-regions in Brazil, in 2010-2011, with coverage being higher in those with smaller wealth inequities and better access to health care.
\end{abstract}

Health Inequalities; Breast Neoplasm; Health Services Accessibility

\author{
Correspondence \\ M. C. Nogueira \\ Faculdade de Medicina, Universidade Federal de Juiz de Fora. \\ Rua Alberto Vieira Lima 357, Juiz de Fora, MG 36050-070, \\ Brasil. \\ mario.cirio.nogueira@gmail.com \\ 1 Faculdade de Medicina, Universidade Federal de Juiz de Fora, \\ Juiz de Fora, Brasil. \\ 2 Department of Medical Statistics, London School of Hygiene \\ and Tropical Medicine, London, UK. \\ 3 Instituto de Medicina Social, Universidade do Estado do Rio de \\ Janeiro, Rio de Janeiro, Brasil.
}




\section{Introduction}

Breast cancer is the most common female cancer worldwide, ranking second as a cause of death due to cancer 1. Mammographic screening programs have been established in many high-income countries since the late 1980s, when results from randomized trials on their effectiveness were first published $2,3,4,5,6$. Although such programs provide the potential to reduce mortality from breast cancer, participation is crucial, with coverage above $70-75 \%$ being regarded as a requirement to achieve such 7,8 .

Several individual-level factors have been shown to affect mammographic screening uptake, including a woman's demographic and socioeconomic characteristics and level of access to health care. Research has also shown that women who reside in socioeconomically deprived areas are less likely to comply with screening 9,10,11,12,13,14,15, even after controlling for individual-level factors $9,11,13$. The characteristics of a woman's area of residence may directly affect her access to screening (e.g., local availability of mammographic screening services), and indirectly through availability of social and material resources (e.g., local breast cancer awareness initiatives, transport networks etc.).

Breast cancer has been the most common female cancer in Brazil since the 1980s 12. It is estimated that a total of 59,700 new cases will be diagnosed yearly in 2018-2019, corresponding to 56.33 cases per 100,000 women-years 16. In all, 16,069 women died from breast cancer in Brazil in 2016, the latest year for which national mortality statistics are available (Departamento de Informática do SUS. http://www.datasus.gov.br). The first guidelines for breast cancer control, which include the recommendation of biannual mammographic screening for women aged 50-69 years, were published by the Brazilian Ministry of Health in 2004 17. National surveys, which include both women with and without private health insurance, showed that mammographic screening coverage has been increasing, with the percentage of women self-reporting a mammographic examination in the previous two years increasing from 54.2\% in 200818 to 60\% in 2013 (Departamento de Informática do SUS. Pesquisa Nacional de Saúde - 2013 - módulo de cobertura de mamografia entre mulheres de 50 anos ou mais. http://tabnet.datasus.gov.br/cgi/deftohtm.exe?pns/pnskb.def, accessed on 02/Sep/2016).

However, these surveys demonstrated marked geographical differences in coverage, pointing to inequities in access to, and uptake of, screening mammography.

The objectives of our study are to describe the spatial distribution of mammographic screening coverage across small geographical areas (i.e., health micro-regions) in Brazil, and to analyze the hypothesis that geographical differences are associated with spatial inequities in socioeconomic conditions, provision of health care, and healthcare services utilization. Health micro-regions are key geographical units in healthcare implementation and provision in Brazil, since each is responsible for coordinating the planning and implementation of healthcare activities and services across several municipalities. Our findings will inform the development and implementation of locally-tailored policies aimed at tackling inequities between the health micro-regions in access to mammographic screening in Brazil.

\section{Methods}

\section{Study design and units of analysis}

An area-based ecological study was conducted in which the units of analysis were the 438 Brazilian health micro-regions (population sizes: $\sim 21,000$ to $>11$ million). Their boundaries were established by the Brazilian Administrative Directive, in accordance with a 2011 federal law which defines a health micro-region as a group of neighboring municipalities with similar socioeconomic features and centralized planning and provision of healthcare services 19.

\section{Outcome}

The outcome of interest was mammographic screening coverage. Since the Brazilian Unified National Health System (SUS) does not collect information on either the number of women screened or the number of women eligible for screening in each year, we estimated, according to previous studies 20 , 
the yearly average number of mammographies performed in 2010-2011 ( $\mathrm{N}=3,432,090)$ in SUS among women aged 50-69 years divided by half of the number of women living in the same health micro-region and of similar age, who depended uniquely on SUS (i.e., who did not have private health insurance) during the same period (Supplementary Table 1: http://cadernos.ensp.fiocruz.br/site/ public_site/arquivo/suppl-e00099817_4234.pdf).

\section{Exposures}

Explanatory variables of interest were grouped according to whether they were distal or proximal in the chain leading from socioeconomic conditions to mammography coverage, according to our substantive model depicted in Figure 1. To represent socioeconomic conditions (deemed as distal variables) specific to each unit of analysis, the following variables were analyzed: population size (log transformed), average Gross Domestic Product (GDP)/per capita (x 1,000 BRL), Gini index of per capita household income (in \%, ranging from 0 , if perfect equality, to 100, if maximal inequality), high education rate (i.e., percentage of the population with secondary or higher education), and urbanization rate (\%). For the provision of health care - the intermediate variables - we considered: percentage of the population registered in the Family Health Strategy (FHS), number of physicians/1,000 inhabitants, number of radiologists/100,000 inhabitants, and number of available mammography machines/10,000 inhabitants. To describe healthcare services utilization, the most proximal dimension, we used number of yearly mammograms per mammography machine. All variables refer to the year 2010 and are available from the Brazilian Health Informatics Department system (DATASUS. http://www.datasus.gov.br) (Supplementary Table 1: http://cadernos.ensp.fiocruz.br/site/public_ site/arquivo/suppl-e00099817_4234.pdf).

\section{Confounders/effect modifiers}

Geographical region, as defined by the Brazilian Institute of Geography and Statistics (IBGE) 21 i.e., Southeast (deemed here as the reference category), South, Northeast, Central, and North -, was included in all analyses to control for factors that were associated with both the exposures and the average yearly number of mammographies, not considered by the selected exposures.

\section{Figure 1}

Conceptual model of area-based associations between socioeconomic conditions, provision of health care, and healthcare services utilization and mammographic screening coverage.

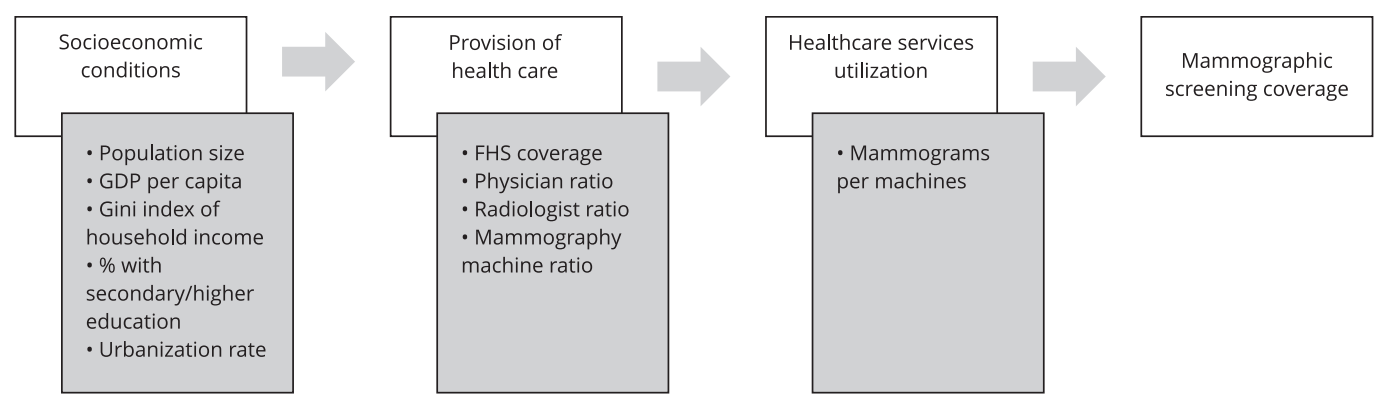

FHS: Family Health Strategy; GDP: Gross Domestic Product. 


\section{Statistical methods}

After initial descriptive and exploratory spatial analyses, four types of regression models were fitted to explain variations in mammographic coverage: simple linear regression; multiple linear regression; multiple linear regression adjusted for spatial autocorrelation; and spatial regimes models, which were also adjusted for spatial autocorrelation 22. Spatial regression models were necessary to control for spatial dependence, which emerges from the interaction of agents across micro-regions, and spatial heterogeneity, which occurs when neighboring micro-regions vary according to the effect of the exposure of interest (e.g., socioeconomic status) on the outcome (i.e., screening coverage) because of interactions with other (observable or not) ecological factors (e.g., political or geographical aspects). If these are disregarded, inferences are likely to be biased.

For each model, we followed the hierarchical step approach proposed by Victora et al. 23 when including additional explanatory variables, each step with inclusion of a group of exposures, from distal to proximal.

For each fitted regression model, predicted residuals were investigated to detect evidence of heteroscedasticity and/or spatial autocorrelation. Linear regression models were fitted using ordinary least squares (OLS) estimation, whereas spatial regression models were estimated using maximum likelihood (ML) estimation, which results in asymptotically normally distributed parameter estimates with large samples and regular spatial weight matrix 24. The spatial auto-regressive (SAR) model, which includes a spatial lag of the dependent variable as an explanatory variable, in addition to all other explanatory variables, was found to fit the data better than the alternative regression models, according to the Lagrange multiplier test 25 . Nested models were compared using the Akaike information criterion (AIC), with smaller values indicating better fit.

Both components of the impact coefficient were estimated: direct, describing the effect on the micro-region itself, and indirect, concerning the effect on its neighboring micro-regions. The total impact corresponds to their sum 26.

Formal comparisons of the regimes and non-regimes spatial models were carried out using the spatial Chow test 27; such test assesses the significance of regional dependences in the model coefficients.

Analyses were carried out using the geographical information system TerraView 4.2 .2 (http:// www.dpi.inpe.br/terraview/index.php), TabWin 3.2 (http://www2.datasus.gov.br/DATASUS/index. php?area=060805\&item=3), R 3.2.2 (https://www.r-project.org/), and R Studio 0.99 (https://www. rstudio.com/).

\section{Results}

In Table 1 and Figure 2 we show the distributions of the exposure and outcome variables per health micro-region and geographical region in Brazil. There was marked variability in mammographic screening coverage across the 438 Brazilian micro-regions, in 2010-2011 (median = 21.6\%; interquartile range (IQR): $8.1 \%-37.9 \%$ ) (Table 1). This variability was present both within and between geographical regions, with micro-regions in the South region having, on average, a 6-fold higher mammographic coverage than those in the North region.

There was also wide variability in the exposure variables across micro-regions. Micro-regions in the North and Northeast had, on average, the lowest average population size, GDP per capita, rate of urbanization, and percentage of the population with secondary or higher educational level, but the largest inequities in household income as observed by the Gini index (Table 1). Micro-regions in the North and Northeast also had, on average, the highest FHS coverage, but the lowest numbers of physicians, radiologists, and mammographic machines per population. Notably, micro-regions in the South and Southeast had not only the highest numbers of mammography machines per population, but also the highest utilization levels (as perceived by the number of mammograms performed per machine). The average number of machines available in micro-regions in the Central region was similar to those in the South and Southeast, but the level of utilization was much lower (Table 1).

Predicted residuals from fitted multiple linear regression models that included geographical region showed significant spatial dependence. Hence, alternative spatial regression models were 
Table 1

Distribution of mammographic screening coverage among women aged 50-69 years and of potential explanatory variables, per health micro-region in Brazil as a whole, and in each of its five geographical regions, 2010-2011.

\begin{tabular}{|c|c|c|c|c|c|c|c|c|}
\hline \multirow[t]{2}{*}{ Variable */Brazilian regions ** } & \multirow{2}{*}{$\begin{array}{l}\text { Number of } \\
\text { health micro- } \\
\text { regions }\end{array}$} & \multicolumn{7}{|c|}{ Variable distribution per health micro-regions } \\
\hline & & Mean & SD & Minimum value & Q1 & Median & Q3 & Maximum value \\
\hline \multicolumn{9}{|l|}{ Outcome variable } \\
\hline \multirow{2}{*}{\multicolumn{9}{|c|}{$\begin{array}{l}\text { Mammography ratio (per } 100 \text { women, } \\
\% \text { ), 2010-2011 }\end{array}$}} \\
\hline & & & & & & & & \\
\hline Brazil & 438 & 24.15 & 17.96 & 0.05 & 8.06 & 21.58 & 37.87 & 84.15 \\
\hline Southeast & 153 & 37.04 & 14.81 & 2.19 & 27.72 & 37.26 & 44.66 & 84.15 \\
\hline South & 68 & 37.19 & 12.81 & 13.95 & 27.92 & 36.52 & 43.02 & 73.58 \\
\hline Northeast & 133 & 13.40 & 10.70 & 0.05 & 5.94 & 12.15 & 17.48 & 51.55 \\
\hline Central & 39 & 8.55 & 8.57 & 0.19 & 2.82 & 5.03 & 12.32 & 32.56 \\
\hline North & 45 & 5.95 & 6.72 & 0.20 & 0.80 & 4.20 & 9.68 & 30.31 \\
\hline \multicolumn{9}{|l|}{$\begin{array}{l}\text { Socioeconomic conditions (distal } \\
\text { variables) }\end{array}$} \\
\hline \multicolumn{9}{|l|}{ Population size (x 1,000), 2010} \\
\hline Brazil & 438 & 435.52 & 846.80 & 21.47 & 152.19 & 250.12 & 396.32 & 11253.50 \\
\hline Southeast & 153 & 525.26 & 1272.24 & 44.27 & 128.29 & 236.19 & 393.43 & 11253.50 \\
\hline South & 68 & 402.75 & 466.20 & 94.96 & 182.14 & 271.87 & 397.34 & 3223.84 \\
\hline Northeast & 133 & 399.11 & 501.23 & 44.66 & 187.46 & 277.16 & 400.62 & 3908.76 \\
\hline Central & 39 & 360.46 & 507.38 & 21.47 & 102.66 & 161.29 & 354.36 & 2570.16 \\
\hline North & 45 & 352.54 & 421.38 & 41.34 & 137.53 & 214.88 & 377.47 & 2119.74 \\
\hline \multicolumn{9}{|l|}{ GDP per capita (x 1,000 BRL), 2010} \\
\hline Brazil & 438 & 14.62 & 9.48 & 3.15 & 6.73 & 13.04 & 19.17 & 59.22 \\
\hline Southeast & 153 & 19.01 & 10.35 & 4.48 & 12.15 & 17.76 & 23.43 & 59.22 \\
\hline South & 68 & 20.08 & 6.55 & 10.04 & 15.53 & 18.84 & 22.89 & 43.10 \\
\hline Northeast & 133 & 7.14 & 4.19 & 3.15 & 4.77 & 5.80 & 8.08 & 39.49 \\
\hline Central & 39 & 18.01 & 8.81 & 7.08 & 12.99 & 15.59 & 21.86 & 58.33 \\
\hline North & 45 & 10.61 & 5.40 & 3.18 & 6.07 & 10.39 & 12.45 & 30.83 \\
\hline \multicolumn{9}{|l|}{ Gini index of per capita household } \\
\hline \multicolumn{9}{|l|}{ income (\%), 2010} \\
\hline Brazil & 438 & 50.92 & 5.11 & 38.00 & 48.00 & 51.00 & 54.00 & 71.00 \\
\hline Southeast & 153 & 47.85 & 3.47 & 40.00 & 45.00 & 48.00 & 50.00 & 65.00 \\
\hline South & 68 & 47.00 & 4.00 & 38.00 & 44.00 & 47.50 & 50.00 & 54.00 \\
\hline Northeast & 133 & 53.68 & 2.92 & 48.00 & 51.00 & 54.00 & 56.00 & 63.00 \\
\hline Central & 39 & 51.69 & 3.77 & 46.00 & 49.00 & 51.00 & 54.00 & 64.00 \\
\hline North & 45 & 58.40 & 4.28 & 50.00 & 55.00 & 58.00 & 61.00 & 71.00 \\
\hline \multicolumn{9}{|l|}{$\begin{array}{l}\text { Population with secondary or higher } \\
\text { education (\%), } 2010\end{array}$} \\
\hline Brazil & 438 & 46.11 & 10.22 & 20.54 & 36.91 & 46.69 & 53.74 & 70.75 \\
\hline Southeast & 153 & 51.11 & 8.94 & 26.63 & 46.57 & 52.90 & 57.87 & 66.90 \\
\hline South & 68 & 51.38 & 6.10 & 39.79 & 47.66 & 50.08 & 54.66 & 67.74 \\
\hline Northeast & 133 & 37.91 & 8.28 & 26.35 & 32.43 & 35.70 & 40.54 & 70.15 \\
\hline Central & 39 & 48.76 & 6.96 & 38.30 & 43.80 & 46.88 & 52.13 & 70.75 \\
\hline North & 45 & 43.08 & 10.41 & 20.54 & 36.11 & 40.35 & 48.84 & 65.79 \\
\hline
\end{tabular}

(continues) 
Table 1 (continued)

\begin{tabular}{|c|c|c|c|c|c|c|c|c|}
\hline \multirow[t]{2}{*}{ Variable */Brazilian regions ** } & \multirow{2}{*}{$\begin{array}{l}\text { Number of } \\
\text { health micro- } \\
\text { regions }\end{array}$} & \multicolumn{7}{|c|}{ Variable distribution per health micro-regions } \\
\hline & & Mean & SD & Minimum value & Q1 & Median & Q3 & Maximum value \\
\hline \multicolumn{9}{|l|}{ Socioeconomic conditions (distal } \\
\hline \multicolumn{9}{|l|}{ variables) } \\
\hline \multicolumn{9}{|l|}{ Urbanization rate (\%), 2010} \\
\hline Brazil & 438 & 75.16 & 15.43 & 40.00 & 63.00 & 77.00 & 89.00 & 100.00 \\
\hline Southeast & 153 & 84.86 & 12.30 & 42.00 & 80.00 & 89.00 & 94.00 & 100.00 \\
\hline South & 68 & 78.68 & 10.94 & 55.00 & 70.50 & 80.00 & 85.25 & 99.00 \\
\hline Northeast & 133 & 63.47 & 13.18 & 40.00 & 54.00 & 62.00 & 70.00 & 100.00 \\
\hline Central & 39 & 79.87 & 10.91 & 58.00 & 69.00 & 81.00 & 89.50 & 98.00 \\
\hline North & 45 & 67.31 & 13.95 & 43.00 & 57.00 & 65.00 & 77.00 & 98.00 \\
\hline \multicolumn{9}{|l|}{ Provision of health care } \\
\hline \multicolumn{9}{|l|}{ (intermediate variables) } \\
\hline \multicolumn{9}{|l|}{ FHS coverage $(\%)$} \\
\hline Brazil & 438 & 78.42 & 22.65 & 13.01 & 64.92 & 86.50 & 98.11 & 100.00 \\
\hline Southeast & 153 & 66.89 & 25.23 & 13.01 & 47.02 & 68.06 & 90.33 & 100.00 \\
\hline South & 68 & 69.14 & 22.52 & 20.70 & 57.06 & 71.06 & 84.93 & 100.00 \\
\hline Northeast & 133 & 94.53 & 9.62 & 40.63 & 92.76 & 98.13 & 100.00 & 100.00 \\
\hline Central & 39 & 80.66 & 17.12 & 27.69 & 71.97 & 85.84 & 93.33 & 100.00 \\
\hline North & 45 & 82.10 & 14.02 & 43.20 & 75.13 & 83.80 & 91.80 & 100.00 \\
\hline \multicolumn{9}{|l|}{ Physician ratio (per 1,000), 2010} \\
\hline Brazil & 438 & 1.00 & 0.62 & 0.12 & 0.54 & 0.85 & 1.31 & 3.64 \\
\hline Southeast & 153 & 1.35 & 0.63 & 0.30 & 0.93 & 1.24 & 1.64 & 3.64 \\
\hline South & 68 & 1.27 & 0.54 & 0.63 & 0.87 & 1.14 & 1.44 & 2.99 \\
\hline Northeast & 133 & 0.64 & 0.40 & 0.26 & 0.42 & 0.53 & 0.68 & 2.69 \\
\hline Central & 39 & 0.92 & 0.54 & 0.42 & 0.58 & 0.85 & 0.96 & 2.73 \\
\hline North & 45 & 0.59 & 0.38 & 0.12 & 0.33 & 0.47 & 0.72 & 1.47 \\
\hline \multicolumn{9}{|l|}{ Radiologist ratio (per 100,000), 2010} \\
\hline Brazil & 438 & 2.93 & 2.59 & 0.00 & 1.00 & 2.00 & 4.00 & 20.00 \\
\hline Southeast & 153 & 3.94 & 2.92 & 0.00 & 2.00 & 3.00 & 5.00 & 20.00 \\
\hline South & 68 & 3.91 & 2.28 & 1.00 & 2.00 & 3.50 & 5.00 & 10.00 \\
\hline Northeast & 133 & 1.79 & 1.85 & 0.00 & 1.00 & 1.00 & 2.00 & 11.00 \\
\hline Central & 39 & 2.87 & 2.48 & 0.00 & 1.00 & 2.00 & 4.00 & 11.00 \\
\hline North & 45 & 1.38 & 1.51 & 0.00 & 0.00 & 1.00 & 2.00 & 5.00 \\
\hline \multicolumn{9}{|l|}{ Mammography machines ratio (per } \\
\hline \multicolumn{9}{|l|}{$10,000), 2010$} \\
\hline Brazil & 438 & 1.19 & 0.86 & 0.00 & 0.63 & 1.14 & 1.66 & 7.70 \\
\hline Southeast & 153 & 1.44 & 0.78 & 0.00 & 0.89 & 1.43 & 1.86 & 3.87 \\
\hline South & 68 & 1.38 & 0.59 & 0.39 & 0.89 & 1.32 & 1.76 & 3.29 \\
\hline Northeast & 133 & 0.84 & 0.70 & 0.00 & 0.43 & 0.71 & 1.18 & 3.31 \\
\hline Central & 39 & 1.31 & 0.96 & 0.00 & 0.66 & 1.38 & 1.95 & 3.67 \\
\hline North & 45 & 0.95 & 1.31 & 0.00 & 0.00 & 0.73 & 1.36 & 7.70 \\
\hline \multicolumn{9}{|l|}{ Healthcare services utilization } \\
\hline \multicolumn{9}{|l|}{ (proximal variable) } \\
\hline \multirow{2}{*}{\multicolumn{9}{|c|}{$\begin{array}{l}\text { Mammographic examinations per } \\
\text { machine (x 100), } 2010\end{array}$}} \\
\hline & & & & & & & & \\
\hline Brazil & 438 & 7.14 & 6.86 & 0.00 & 1.43 & 6.03 & 10.30 & 52.06 \\
\hline Southeast & 153 & 9.66 & 6.58 & 0.00 & 5.38 & 8.21 & 12.48 & 30.39 \\
\hline South & 68 & 11.94 & 6.42 & 3.80 & 7.89 & 9.75 & 13.79 & 34.33 \\
\hline Northeast & 133 & 5.02 & 6.28 & 0.00 & 0.14 & 3.87 & 7.78 & 52.06 \\
\hline Central & 39 & 2.24 & 2.66 & 0.00 & 0.11 & 1.59 & 3.67 & 10.52 \\
\hline North & 45 & 1.82 & 3.06 & 0.00 & 0.00 & 0.22 & 2.41 & 12.42 \\
\hline
\end{tabular}

FHS: Family Health Strategy; GDP: Gross Domestic Product; Q1: 25\% percentiles of the distribution; Q3: 25\% and 75\% percentiles of the distribution; SD: standard deviation.

* See Supplementary Table 1 (http://cadernos.ensp.fiocruz.br/site/public_site/arquivo/suppl-e00099817_4234.pdf) for definition of each variable and its data sources;

** Geographical regions listed in descending order of their average population size. 


\section{Figure 2}

Distribution of mammographic screening coverage among women aged 50-69 years in 2010-2011 by health micro-region $(n=438)$, Brazil.

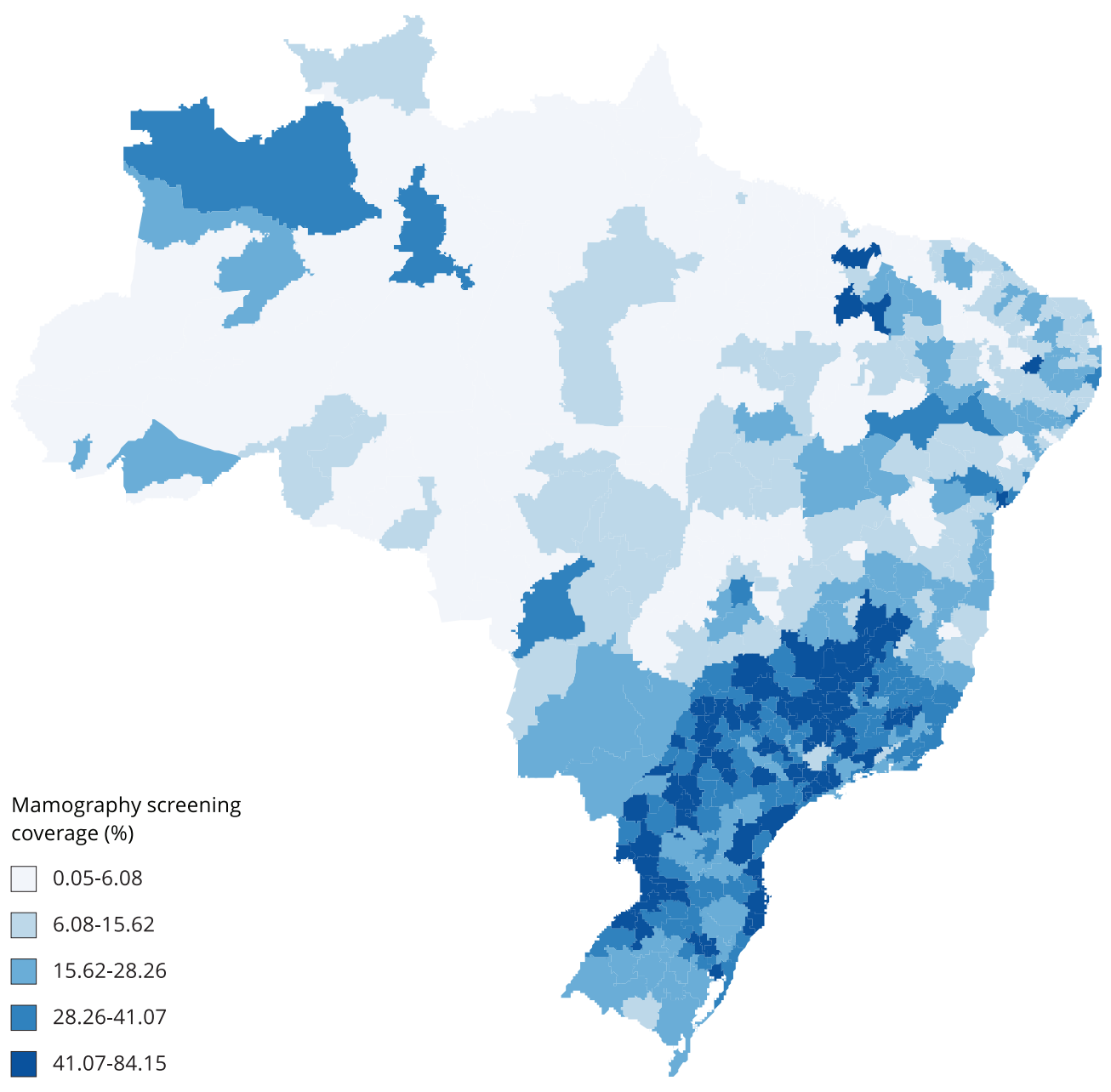

considered. The results from fitting the best spatial model, SAR, are presented in Table 2. The first step involved jointly modelling all distal explanatory variables (i.e., socioeconomic indicators) as well as geographical regions. Because of collinearities with other variables, high education rate was not kept in the model. Both population size (direct impact $=1.985$; indirect impact $=1.251$ ) and degree of urbanization (direct impact $=0.158$; indirect impact $=0.099$ ) were positively associated with mammography coverage, whereas the Gini index (direct impact $=-0.648$; indirect impact $=-0.408$ ) was inversely associated with mammographic coverage, with the magnitude of direct impacts being higher than the magnitude of the indirect ones (via neighboring micro-regions). These impacts mean that, for example, a $1 \%$ increase in the Gini index was associated with a $0.6 \%$ decrease in mammographic coverage in a given micro-region, and a $0.4 \%$ decrease in its neighboring micro-regions, maintaining the other socioeconomic indicators constant. On the other hand, a $1 \%$ increase in the urbanization rate was associated with a $0.2 \%$ increase in coverage in a given micro-region, as well as a $0.1 \%$ increase in its neighboring micro-regions, again maintaining the other variables constant. There were also significant estimated differences in mammographic coverage between geographical 
Table 2

Correlates of mammographic screening coverage (\%) at the health micro-region level. Brazil, 2010-2011.

\begin{tabular}{|c|c|c|c|c|c|c|c|c|c|}
\hline \multirow[t]{2}{*}{ Variables * } & \multicolumn{3}{|c|}{$\begin{array}{c}\text { Step } 1 \\
\text { Impacts (p-value) }\end{array}$} & \multicolumn{3}{|c|}{$\begin{array}{c}\text { Step } 2 \\
\text { Impacts ( } p \text {-value) }\end{array}$} & \multicolumn{3}{|c|}{$\begin{array}{c}\text { Step } 3 \\
\text { Impacts (p-value) }\end{array}$} \\
\hline & Direct & Indirect & Total & Direct & Indirect & Total & Direct & Indirect & Total \\
\hline \multicolumn{10}{|l|}{ Regions ** } \\
\hline Southeast & Ref. & Ref. & Ref. & Ref. & Ref. & Ref. & Ref. & Ref. & Ref. \\
\hline South & $\begin{array}{c}0.263 \\
(0.859)\end{array}$ & $\begin{array}{c}0.166 \\
(0.863)\end{array}$ & $\begin{array}{c}0.429 \\
(0.860)\end{array}$ & $\begin{array}{l}-0.103 \\
(0.850)\end{array}$ & $\begin{array}{l}-0.065 \\
(0.859)\end{array}$ & $\begin{array}{l}-0.167 \\
(0.853)\end{array}$ & $\begin{array}{l}-2.006 \\
(0.100)\end{array}$ & $\begin{array}{l}-0.859 \\
(0.142)\end{array}$ & $\begin{array}{l}-2.865 \\
(0.108)\end{array}$ \\
\hline Northeast & $\begin{array}{l}-6.732 \\
(0.001)\end{array}$ & $\begin{array}{l}-4.241 \\
(0.001)\end{array}$ & $\begin{array}{l}-10.972 \\
(<0.001)\end{array}$ & $\begin{array}{c}-6.793 \\
(<0.001)\end{array}$ & $\begin{array}{c}-4.274 \\
(<0.001)\end{array}$ & $\begin{array}{l}-11.067 \\
(<0.001)\end{array}$ & $\begin{array}{l}-2.676 \\
(0.078)\end{array}$ & $\begin{array}{l}-1.145 \\
(0.089)\end{array}$ & $\begin{array}{l}-3.820 \\
(0.075)\end{array}$ \\
\hline Central & $\begin{array}{l}-14.693 \\
(<0.001)\end{array}$ & $\begin{array}{c}-9.257 \\
(<0.001)\end{array}$ & $\begin{array}{l}-23.951 \\
(<0.001)\end{array}$ & $\begin{array}{l}-14.811 \\
(<0.001)\end{array}$ & $\begin{array}{c}-9.319 \\
(<0.001)\end{array}$ & $\begin{array}{l}-24.130 \\
(<0.001)\end{array}$ & $\begin{array}{c}-9.846 \\
(<0.001)\end{array}$ & $\begin{array}{c}-4.214 \\
(<0.001)\end{array}$ & $\begin{array}{l}-14.060 \\
(<0.001)\end{array}$ \\
\hline North & $\begin{array}{l}-8.500 \\
(0.001)\end{array}$ & $\begin{array}{l}-5.355 \\
(0.002)\end{array}$ & $\begin{array}{l}-13.856 \\
(0.001)\end{array}$ & $\begin{array}{l}-7.831 \\
(0.001)\end{array}$ & $\begin{array}{l}-4.927 \\
(0.001)\end{array}$ & $\begin{array}{l}-12.758 \\
(0.000)\end{array}$ & $\begin{array}{l}-2.641 \\
(0.237)\end{array}$ & $\begin{array}{l}-1.131 \\
(0.261)\end{array}$ & $\begin{array}{l}-3.772 \\
(0.237)\end{array}$ \\
\hline Population (x 1,000, log) & $\begin{array}{c}1.985 \\
(0.005)\end{array}$ & $\begin{array}{c}1.251 \\
(0.016)\end{array}$ & $\begin{array}{c}3.236 \\
(0.006)\end{array}$ & $\begin{array}{c}1.668 \\
(0.039)\end{array}$ & $\begin{array}{c}1.050 \\
(0.058)\end{array}$ & $\begin{array}{c}2.718 \\
(0.042)\end{array}$ & $\begin{array}{l}-0.278 \\
(0.734)\end{array}$ & $\begin{array}{l}-0.119 \\
(0.738)\end{array}$ & $\begin{array}{l}-0.396 \\
(0.734)\end{array}$ \\
\hline GDP per capita (x 1,000 BRL) & $\begin{array}{c}0.102 \\
(0.106)\end{array}$ & $\begin{array}{c}0.064 \\
(0.115)\end{array}$ & $\begin{array}{c}0.166 \\
(0.106)\end{array}$ & $\begin{array}{c}0.101 \\
(0.144)\end{array}$ & $\begin{array}{c}0.064 \\
(0.150)\end{array}$ & $\begin{array}{c}0.165 \\
(0.142)\end{array}$ & $\begin{array}{c}0.112 \\
(0.152)\end{array}$ & $\begin{array}{c}0.048 \\
(0.188)\end{array}$ & $\begin{array}{c}0.160 \\
(0.156)\end{array}$ \\
\hline Gini index (\%) & $\begin{array}{c}-0.648 \\
(<0.001)\end{array}$ & $\begin{array}{l}-0.408 \\
(0.002)\end{array}$ & $\begin{array}{l}-1.056 \\
(0.000)\end{array}$ & $\begin{array}{l}-0.600 \\
(0.000)\end{array}$ & $\begin{array}{l}-0.378 \\
(0.000)\end{array}$ & $\begin{array}{l}-0.978 \\
(0.000)\end{array}$ & $\begin{array}{c}-0.562 \\
(<0.001)\end{array}$ & $\begin{array}{c}-0.241 \\
(<0.001)\end{array}$ & $\begin{array}{c}-0.803 \\
(<0.001)\end{array}$ \\
\hline Urbanization rate (\%) & $\begin{array}{c}0.158 \\
(0.002)\end{array}$ & $\begin{array}{c}0.099 \\
(0.009)\end{array}$ & $\begin{array}{c}0.257 \\
(0.002)\end{array}$ & $\begin{array}{c}0.074 \\
(0.363)\end{array}$ & $\begin{array}{c}0.046 \\
(0.382)\end{array}$ & $\begin{array}{c}0.120 \\
(0.368)\end{array}$ & $\begin{array}{c}0.037 \\
(0.421)\end{array}$ & $\begin{array}{c}0.016 \\
(0.457)\end{array}$ & $\begin{array}{c}0.052 \\
(0.429)\end{array}$ \\
\hline FHS coverage $(\%)$ & & & & $\begin{array}{c}0.049 \\
(0.120)\end{array}$ & $\begin{array}{c}0.031 \\
(0.126)\end{array}$ & $\begin{array}{c}0.080 \\
(0.117)\end{array}$ & $\begin{array}{l}-0.004 \\
(0.805)\end{array}$ & $\begin{array}{l}-0.002 \\
(0.815)\end{array}$ & $\begin{array}{l}-0.006 \\
(0.807)\end{array}$ \\
\hline $\begin{array}{l}\text { Radiologists ratio per } 100,000 \\
\text { inhabitants }\end{array}$ & & & & $\begin{array}{c}0.870 \\
(0.001)\end{array}$ & $\begin{array}{c}0.548 \\
(0.011)\end{array}$ & $\begin{array}{c}1.418 \\
(0.002)\end{array}$ & $\begin{array}{c}0.919 \\
(<0.001)\end{array}$ & $\begin{array}{c}0.393 \\
(0.001)\end{array}$ & $\begin{array}{c}1.312 \\
(<0.001)\end{array}$ \\
\hline $\begin{array}{l}\text { Mammography machines ratio per } \\
10,000 \text { inhabitants }\end{array}$ & & & & $\begin{array}{c}2.451 \\
(<0.001)\end{array}$ & $\begin{array}{c}1.542 \\
(0.001)\end{array}$ & $\begin{array}{c}3.993 \\
(<0.001)\end{array}$ & $\begin{array}{c}3.715 \\
(<0.001)\end{array}$ & $\begin{array}{c}1.590 \\
(<0.001)\end{array}$ & $\begin{array}{c}5.305 \\
(<0.001)\end{array}$ \\
\hline $\begin{array}{l}\text { Mammograms per mammography } \\
\text { machine }(x 100)\end{array}$ & & & & & & & $\begin{array}{c}0.948 \\
(<0.001)\end{array}$ & $\begin{array}{c}0.406 \\
(<0.001)\end{array}$ & $\begin{array}{c}1.353 \\
(<0.001)\end{array}$ \\
\hline Model diagnostics & & & & & & & & & \\
\hline $\mathrm{AIC}$ & 3302.700 & & & 3278.900 & & & 3140.200 & & \\
\hline Breusch-Pagan test heterocedasticity & 0.106 & & & 0.167 & & & 0.290 & & \\
\hline Test for residual autocorrelation & 0.396 & & & 0.460 & & & 0.072 & & \\
\hline
\end{tabular}

AIC: Akaike information criterion; FHS: Family Health Strategy; GDP: Gross Domestic Product; Ref.: reference.

* See Supplementary Table 1 (http://cadernos.ensp.fiocruz.br/site/public_site/arquivo/suppl-e00099817_4234.pdf) for definition of each variable and its data sources;

** Regions listed in descending order of their average population size.

regions, despite having controlled for the other distal explanatory variables. Relative to micro-regions in the Southeast, mammographic coverage was, on average, 24\% lower in those in the Central, 13.9\% lower in those in the North, and $11 \%$ lower in those in the Northeast.

The second step involved jointly modelling distal and intermediate (i.e., provision of health care) variables. Because of collinearity, physician ratio was not included in these analyses. Population size remained with its positive association, and the Gini index, its negative association, with mammographic coverage, whereas urbanization rate was no longer associated with the outcome. Among the variables of provision of healthcare services, only number of radiologists and number of machines per population were positively associated with the outcome, with their direct impacts being higher than their indirect ones. Micro-regions in the Central, North, and Northeast had, as in step 1, lower average mammography coverage than those in the Southeast, with little changes in the magnitude of the estimates (Table 2). 
Finally, a positive association was found between mammographic coverage and healthcare services utilization - as observed by the number of mammograms per machine - when this variable was jointly modelled with the distal and provision of healthcare services variables (step 3) (Table 2). Both the number of radiologists and the number of mammographic machines per population remained positively associated with the outcome in such model. However, the Gini index was the only distal variable that remained associated (inversely) with mammographic coverage, although with a slightly smaller estimated impact than in step 2. In this more comprehensive model, only micro-regions in the Central region had significantly lower mammographic coverage than those in the Southeast (reference).

Results of the spatial regime analysis, in which the SAR model was expanded to allow for regionspecific coefficients, i.e., interactions with the region, are shown in Table 3. As before, the threestep hierarchical approach was implemented. There was evidence of heterogeneity of results across regions, which was corroborated by the spatial Chow test, between the models fitted at each step, and its equivalent model of spatial regimes. In the wealthiest regions, namely South and Southeast, accounting for intermediate and proximal variables, mammographic coverage was associated with only a few specific socioeconomic indicators (i.e., inversely with the Gini index in both regions and positively with population size only in the South), but (positively) associated with all provision of health care and healthcare services utilization indicators (with the exception of FHS coverage) (Table 3). In the Central and North regions, coverage was (positively) associated only with provision of health care indicators (i.e., number of radiologists/population in Central; number of mammography machines/population in the North) and the healthcare services utilization variable (Table 3). Finally, in the Northeast, the healthcare services utilization variable (mammograms/machine) was the only variable found to be associated (positively) with the outcome (Table 3).

Table 3

Correlates of mammographic screening coverage at the health micro-region level, stratified by geographical region *.

\begin{tabular}{|c|c|c|c|c|c|c|c|c|c|}
\hline \multirow[t]{2}{*}{ Regions/Variables } & \multicolumn{3}{|c|}{$\begin{array}{c}\text { Step } 1 \\
\text { Impacts ( } p \text {-value) }\end{array}$} & \multicolumn{3}{|c|}{$\begin{array}{c}\text { Step } 2 \\
\text { Impacts (p-value) }\end{array}$} & \multicolumn{3}{|c|}{$\begin{array}{c}\text { Step } 3 \\
\text { Impacts ( } p \text {-value) }\end{array}$} \\
\hline & Direct & Indirect & Total & Direct & Indirect & Total & Direct & Indirect & Total \\
\hline \multicolumn{10}{|l|}{ Souheast } \\
\hline Population (x 1,000, log) & $\begin{array}{c}0.848 \\
(0.395)\end{array}$ & $\begin{array}{c}0.450 \\
(0.408)\end{array}$ & $\begin{array}{c}1.298 \\
(0.394)\end{array}$ & $\begin{array}{c}0.878 \\
(0.562)\end{array}$ & $\begin{array}{c}0.444 \\
(0.569)\end{array}$ & $\begin{array}{c}1.323 \\
(0.561)\end{array}$ & $\begin{array}{l}-1.566 \\
(0.114)\end{array}$ & $\begin{array}{l}-0.489 \\
(0.154)\end{array}$ & $\begin{array}{l}-2.055 \\
(0.115)\end{array}$ \\
\hline GDP per capita (x 1,000 BRL) & $\begin{array}{c}0.146 \\
(0.229)\end{array}$ & $\begin{array}{c}0.077 \\
(0.296)\end{array}$ & $\begin{array}{c}0.224 \\
(0.247)\end{array}$ & $\begin{array}{c}0.153 \\
(0.137)\end{array}$ & $\begin{array}{c}0.077 \\
(0.191)\end{array}$ & $\begin{array}{c}0.231 \\
(0.147)\end{array}$ & $\begin{array}{c}0.144 \\
(0.129)\end{array}$ & $\begin{array}{c}0.045 \\
(0.162)\end{array}$ & $\begin{array}{c}0.189 \\
(0.127)\end{array}$ \\
\hline Gini index (\%) & $\begin{array}{l}-1.416 \\
(<0.001)\end{array}$ & $\begin{array}{c}-0.751 \\
(<0.001)\end{array}$ & $\begin{array}{c}-2.168 \\
(<0.001)\end{array}$ & $\begin{array}{c}-1.373 \\
(<0.001)\end{array}$ & $\begin{array}{l}-0.695 \\
(0.001)\end{array}$ & $\begin{array}{c}-2.067 \\
(<0.001)\end{array}$ & $\begin{array}{c}-0.914 \\
(<0.001)\end{array}$ & $\begin{array}{l}-0.285 \\
(0.003)\end{array}$ & $\begin{array}{c}-1.199 \\
(<0.001)\end{array}$ \\
\hline Urbanization rate (\%) & $\begin{array}{l}-0.052 \\
(0.230)\end{array}$ & $\begin{array}{c}0.071 \\
(0.250)\end{array}$ & $\begin{array}{c}0.205 \\
(0.230)\end{array}$ & $\begin{array}{c}0.030 \\
(0.614)\end{array}$ & $\begin{array}{c}0.015 \\
(0.675)\end{array}$ & $\begin{array}{c}0.045 \\
(0.632)\end{array}$ & $\begin{array}{c}0.030 \\
(0.919)\end{array}$ & $\begin{array}{c}0.009 \\
(0.924)\end{array}$ & $\begin{array}{c}0.039 \\
(0.919)\end{array}$ \\
\hline FHS coverage $(\%)$ & & & & $\begin{array}{c}0.008 \\
(0.906)\end{array}$ & $\begin{array}{c}0.004 \\
(0.900)\end{array}$ & $\begin{array}{c}0.013 \\
(0.903)\end{array}$ & $\begin{array}{l}-0.061 \\
(0.048)\end{array}$ & $\begin{array}{l}-0.019 \\
(0.083)\end{array}$ & $\begin{array}{l}-0.080 \\
(0.050)\end{array}$ \\
\hline $\begin{array}{l}\text { Radiologists ratio per } 100,000 \\
\text { inhabitants }\end{array}$ & & & & $\begin{array}{c}0.598 \\
(0.063)\end{array}$ & $\begin{array}{c}0.303 \\
(0.089)\end{array}$ & $\begin{array}{c}0.901 \\
(0.064)\end{array}$ & $\begin{array}{c}0.692 \\
(0.014)\end{array}$ & $\begin{array}{c}0.216 \\
(0.049)\end{array}$ & $\begin{array}{c}0.908 \\
(0.015)\end{array}$ \\
\hline $\begin{array}{l}\text { Mammography machines ratio per } \\
10,000 \text { inhabitants }\end{array}$ & & & & $\begin{array}{c}2.300 \\
(0.008)\end{array}$ & $\begin{array}{c}1.432 \\
(0.031)\end{array}$ & $\begin{array}{c}4.262 \\
(0.011)\end{array}$ & $\begin{array}{c}5.510 \\
(<0.001)\end{array}$ & $\begin{array}{c}1.719 \\
(0.001)\end{array}$ & $\begin{array}{c}7.229 \\
(<0.001)\end{array}$ \\
\hline $\begin{array}{l}\text { Mammograms per mammography } \\
\text { machine (x 100) }\end{array}$ & & & & & & & $\begin{array}{c}0.965 \\
(<0.001)\end{array}$ & $\begin{array}{c}0.301 \\
(0.001)\end{array}$ & $\begin{array}{c}1.266 \\
(<0.001)\end{array}$ \\
\hline
\end{tabular}

(continues) 
Table 3 (continued)

\begin{tabular}{|c|c|c|c|c|c|c|c|c|c|}
\hline \multirow[t]{2}{*}{ Regions/Variables } & \multicolumn{3}{|c|}{$\begin{array}{c}\text { Step } 1 \\
\text { Impacts ( } p \text {-value) }\end{array}$} & \multicolumn{3}{|c|}{$\begin{array}{c}\text { Step } 2 \\
\text { Impacts ( } p \text {-value) }\end{array}$} & \multicolumn{3}{|c|}{$\begin{array}{c}\text { Step } 3 \\
\text { Impacts ( } p \text {-value) }\end{array}$} \\
\hline & Direct & Indirect & Total & Direct & Indirect & Total & Direct & Indirect & Total \\
\hline \multicolumn{10}{|l|}{ South } \\
\hline Population (x 1,000, log) & $\begin{array}{l}9.210 \\
(0.000)\end{array}$ & $\begin{array}{c}4.885 \\
(0.011)\end{array}$ & $\begin{array}{l}14.095 \\
(0.000)\end{array}$ & $\begin{array}{l}7.710 \\
(0.004)\end{array}$ & $\begin{array}{c}3.902 \\
(0.027)\end{array}$ & $\begin{array}{l}11.613 \\
(0.006)\end{array}$ & $\begin{array}{c}5.946 \\
(<0.001)\end{array}$ & $\begin{array}{c}1.856 \\
(0.012)\end{array}$ & $\begin{array}{c}7.802 \\
(<0.001)\end{array}$ \\
\hline GDP per capita (x 1,000 BRL) & $\begin{array}{l}-0.018 \\
(0.975)\end{array}$ & $\begin{array}{l}-0.010 \\
(0.966)\end{array}$ & $\begin{array}{l}-0.027 \\
(0.972)\end{array}$ & $\begin{array}{c}0.025 \\
(0.991)\end{array}$ & $\begin{array}{c}0.013 \\
(1.000)\end{array}$ & $\begin{array}{c}0.038 \\
(0.994)\end{array}$ & $\begin{array}{c}0.101 \\
(0.698)\end{array}$ & $\begin{array}{c}0.031 \\
(0.726)\end{array}$ & $\begin{array}{c}0.132 \\
(0.703)\end{array}$ \\
\hline Gini index (\%) & $\begin{array}{l}-1.275 \\
(0.000)\end{array}$ & $\begin{array}{l}-0.676 \\
(0.006)\end{array}$ & $\begin{array}{l}-1.951 \\
(0.000)\end{array}$ & $\begin{array}{l}-0.872 \\
(0.011)\end{array}$ & $\begin{array}{l}-0.441 \\
(0.028)\end{array}$ & $\begin{array}{l}-1.313 \\
(0.012)\end{array}$ & $\begin{array}{l}-0.899 \\
(0.002)\end{array}$ & $\begin{array}{l}-0.280 \\
(0.008)\end{array}$ & $\begin{array}{l}-1.179 \\
(0.001)\end{array}$ \\
\hline Urbanization rate (\%) & $\begin{array}{l}-0.223 \\
(0.158)\end{array}$ & $\begin{array}{l}-0.118 \\
(0.216)\end{array}$ & $\begin{array}{l}-0.341 \\
(0.169)\end{array}$ & $\begin{array}{l}-0.058 \\
(0.737)\end{array}$ & $\begin{array}{l}-0.029 \\
(0.734)\end{array}$ & $\begin{array}{l}-0.088 \\
(0.734)\end{array}$ & $\begin{array}{l}-0.114 \\
(0.417)\end{array}$ & $\begin{array}{l}-0.035 \\
(0.442)\end{array}$ & $\begin{array}{l}-0.149 \\
(0.418)\end{array}$ \\
\hline FHS coverage (\%) & & & & $\begin{array}{c}0.203 \\
(0.002)\end{array}$ & $\begin{array}{c}0.103 \\
(0.012)\end{array}$ & $\begin{array}{c}0.306 \\
(0.002)\end{array}$ & $\begin{array}{c}0.072 \\
(0.167)\end{array}$ & $\begin{array}{c}0.022 \\
(0.220)\end{array}$ & $\begin{array}{c}0.094 \\
(0.173)\end{array}$ \\
\hline $\begin{array}{l}\text { Radiologists ratio per 100,000 } \\
\text { inhabitants }\end{array}$ & & & & $\begin{array}{l}1.259 \\
(0.072)\end{array}$ & $\begin{array}{c}0.637 \\
(0.117)\end{array}$ & $\begin{array}{c}1.896 \\
(0.080)\end{array}$ & $\begin{array}{c}1.127 \\
(0.037)\end{array}$ & $\begin{array}{l}0.352 \\
(0.101)\end{array}$ & $\begin{array}{c}1.478 \\
(0.043)\end{array}$ \\
\hline $\begin{array}{l}\text { Mammography machines ratio per } \\
10,000 \text { inhabitants }\end{array}$ & & & & $\begin{array}{l}3.871 \\
(0.071)\end{array}$ & $\begin{array}{c}1.959 \\
(0.115)\end{array}$ & $\begin{array}{c}5.830 \\
(0.078)\end{array}$ & $\begin{array}{c}13.215 \\
(<0.001)\end{array}$ & $\begin{array}{c}4.124 \\
(0.001)\end{array}$ & $\begin{array}{c}17.339 \\
(<0.001)\end{array}$ \\
\hline $\begin{array}{l}\text { Mammograms per mammography } \\
\text { machine (x 100) }\end{array}$ & & & & & & & $\begin{array}{c}1.311 \\
(<0.001)\end{array}$ & $\begin{array}{c}0.409 \\
(0.002)\end{array}$ & $\begin{array}{c}1.720 \\
(<0.001)\end{array}$ \\
\hline \multicolumn{10}{|l|}{ Northeast } \\
\hline Population (x 1,000, log) & $\begin{array}{c}3.572 \\
(0.012)\end{array}$ & $\begin{array}{c}1.895 \\
(0.027)\end{array}$ & $\begin{array}{c}5.467 \\
(0.013)\end{array}$ & $\begin{array}{l}2.744 \\
(0.049)\end{array}$ & $\begin{array}{c}1.389 \\
(0.059)\end{array}$ & $\begin{array}{l}4.133 \\
(0.047)\end{array}$ & $\begin{array}{c}0.332 \\
(0.741)\end{array}$ & $\begin{array}{c}0.104 \\
(0.740)\end{array}$ & $\begin{array}{c}0.436 \\
(0.739)\end{array}$ \\
\hline GDP per capita (x 1,000 BRL) & $\begin{array}{l}0.345 \\
(0.156)\end{array}$ & $\begin{array}{c}0.183 \\
(0.207)\end{array}$ & $\begin{array}{l}0.528 \\
(0.167)\end{array}$ & $\begin{array}{l}0.265 \\
(0.272)\end{array}$ & $\begin{array}{l}0.134 \\
(0.301)\end{array}$ & $\begin{array}{l}0.097 \\
(0.276)\end{array}$ & $\begin{array}{l}0.032 \\
(0.956)\end{array}$ & $\begin{array}{l}0.010 \\
(0.958)\end{array}$ & $\begin{array}{l}0.042 \\
(0.956)\end{array}$ \\
\hline Gini index (\%) & $\begin{array}{l}0.154 \\
(0.557)\end{array}$ & $\begin{array}{c}0.082 \\
(0.599)\end{array}$ & $\begin{array}{c}0.236 \\
(0.569)\end{array}$ & $\begin{array}{l}0.106 \\
(0.586)\end{array}$ & $\begin{array}{l}0.054 \\
(0.604)\end{array}$ & $\begin{array}{c}0.160 \\
(0.588)\end{array}$ & $\begin{array}{c}0.079 \\
(0.885)\end{array}$ & $\begin{array}{l}0.025 \\
(0.891)\end{array}$ & $\begin{array}{c}0.103 \\
(0.886)\end{array}$ \\
\hline Urbanization rate (\%) & $\begin{array}{c}0.096 \\
(0.299)\end{array}$ & $\begin{array}{c}0.051 \\
(0.307)\end{array}$ & $\begin{array}{c}0.148 \\
(0.295)\end{array}$ & $\begin{array}{c}0.020 \\
(0.762)\end{array}$ & $\begin{array}{c}0.010 \\
(0.776)\end{array}$ & $\begin{array}{c}0.030 \\
(0.765)\end{array}$ & $\begin{array}{c}0.094 \\
(0.347)\end{array}$ & $\begin{array}{c}0.029 \\
(0.379)\end{array}$ & $\begin{array}{c}0.123 \\
(0.350)\end{array}$ \\
\hline FHS coverage $(\%)$ & & & & $\begin{array}{l}-0.078 \\
(0.570)\end{array}$ & $\begin{array}{l}-0.040 \\
(0.555)\end{array}$ & $\begin{array}{l}-0.118 \\
(0.562)\end{array}$ & $\begin{array}{l}-0.069 \\
(0.476)\end{array}$ & $\begin{array}{l}-0.022 \\
(0.489)\end{array}$ & $\begin{array}{l}-0.091 \\
(0.476)\end{array}$ \\
\hline $\begin{array}{l}\text { Radiologists ratio per } 100,000 \\
\text { inhabitants }\end{array}$ & & & & $\begin{array}{c}0.514 \\
(0.490)\end{array}$ & $\begin{array}{c}0.260 \\
(0.519)\end{array}$ & $\begin{array}{c}0.774 \\
(0.496)\end{array}$ & $\begin{array}{c}0.757 \\
(0.191)\end{array}$ & $\begin{array}{c}0.236 \\
(0.228)\end{array}$ & $\begin{array}{c}0.994 \\
(0.193)\end{array}$ \\
\hline $\begin{array}{l}\text { Mammography machines ratio per } \\
10,000 \text { inhabitants }\end{array}$ & & & & $\begin{array}{l}1.547 \\
(0.192)\end{array}$ & $\begin{array}{c}0.783 \\
(0.202)\end{array}$ & $\begin{array}{c}2.330 \\
(0.188)\end{array}$ & $\begin{array}{c}0.951 \\
(0.336)\end{array}$ & $\begin{array}{c}0.297 \\
(0.382)\end{array}$ & $\begin{array}{c}1.248 \\
(0.340)\end{array}$ \\
\hline $\begin{array}{l}\text { Mammograms per mammography } \\
\text { machine (x 100) }\end{array}$ & & & & & & & $\begin{array}{c}1.036 \\
(0.000)\end{array}$ & $\begin{array}{c}0.323 \\
(0.001)\end{array}$ & $\begin{array}{c}1.360 \\
(0.000)\end{array}$ \\
\hline \multicolumn{10}{|l|}{ Central } \\
\hline Population (x 1,000, log) & $\begin{array}{l}4.946 \\
(0.049)\end{array}$ & $\begin{array}{c}2.623 \\
(0.103)\end{array}$ & $\begin{array}{c}7.569 \\
(0.059)\end{array}$ & $\begin{array}{l}1.574 \\
(0.644)\end{array}$ & $\begin{array}{c}0.797 \\
(0.676)\end{array}$ & $\begin{array}{l}2.371 \\
(0.652)\end{array}$ & $\begin{array}{c}0.575 \\
(0.712)\end{array}$ & $\begin{array}{c}0.180 \\
(0.726)\end{array}$ & $\begin{array}{c}0.755 \\
(0.714)\end{array}$ \\
\hline GDP per capita (x 1,000 BRL) & $\begin{array}{l}-0.008 \\
(0.852)\end{array}$ & $\begin{array}{l}-0.004 \\
(0.877)\end{array}$ & $\begin{array}{l}-0.012 \\
(0.859)\end{array}$ & $\begin{array}{l}-0.218 \\
(0.284)\end{array}$ & $\begin{array}{l}-0.110 \\
(0.314)\end{array}$ & $\begin{array}{l}-0.328 \\
(0.288)\end{array}$ & $\begin{array}{l}-0.277 \\
(0.231)\end{array}$ & $\begin{array}{l}-0.087 \\
(0.265)\end{array}$ & $\begin{array}{l}-0.364 \\
(0.233)\end{array}$ \\
\hline Gini index (\%) & $\begin{array}{l}-0.036 \\
(0.895)\end{array}$ & $\begin{array}{l}-0.019 \\
(0.884)\end{array}$ & $\begin{array}{l}-0.056 \\
(0.891)\end{array}$ & $\begin{array}{l}-0.110 \\
(0.852)\end{array}$ & $\begin{array}{l}-0.056 \\
(0.855)\end{array}$ & $\begin{array}{l}-0.166 \\
(0.852)\end{array}$ & $\begin{array}{l}-0.123 \\
(0.647)\end{array}$ & $\begin{array}{l}-0.038 \\
(0.661)\end{array}$ & $\begin{array}{l}-0.161 \\
(0.648)\end{array}$ \\
\hline Urbanization rate (\%) & $\begin{array}{l}-0.052 \\
(0.883)\end{array}$ & $\begin{array}{c}-0.028 \\
(0.876)\end{array}$ & $\begin{array}{l}-0.079 \\
(0.880)\end{array}$ & $\begin{array}{c}0.024 \\
(0.869)\end{array}$ & $\begin{array}{c}0.012 \\
(0.865)\end{array}$ & $\begin{array}{c}0.035 \\
(0.867)\end{array}$ & $\begin{array}{l}-0.122 \\
(0.536)\end{array}$ & $\begin{array}{l}-0.038 \\
(0.541)\end{array}$ & $\begin{array}{l}-0.160 \\
(0.534)\end{array}$ \\
\hline FHS coverage (\%) & & & & $\begin{array}{l}0.107 \\
(0.425)\end{array}$ & $\begin{array}{c}0.054 \\
(0.441)\end{array}$ & $\begin{array}{l}0.161 \\
(0.427)\end{array}$ & $\begin{array}{l}0.050 \\
(0.690)\end{array}$ & $\begin{array}{l}0.015 \\
(0.672)\end{array}$ & $\begin{array}{c}0.062 \\
(0.683)\end{array}$ \\
\hline Radiologists ratio per 100,000 & & & & 2.767 & 1.400 & 4.167 & 2.293 & 0.716 & 3.008 \\
\hline inhabitants & & & & $(0.003)$ & $(0.020)$ & $(0.004)$ & $(0.018)$ & $(0.074)$ & $(0.024)$ \\
\hline Mammography machines ratio per & & & & 0.624 & 0.316 & 0.940 & 0.968 & 0.302 & 1.270 \\
\hline 10,000 inhabitants & & & & $(0.810)$ & $(0.797)$ & $(0.805)$ & $(0.434)$ & $(0.434)$ & $(0.430)$ \\
\hline Mammograms per mammography & & & & & & & 1.848 & 0.577 & 2.425 \\
\hline machine $(x$ 100) & & & & & & & $(0.009)$ & $(0.033)$ & $(0.009)$ \\
\hline
\end{tabular}

(continues) 
Table 3 (continued)

\begin{tabular}{|c|c|c|c|c|c|c|c|c|c|}
\hline \multirow[t]{2}{*}{ Regions/Variables } & \multicolumn{3}{|c|}{$\begin{array}{c}\text { Step } 1 \\
\text { Impacts ( } p \text {-value })\end{array}$} & \multicolumn{3}{|c|}{$\begin{array}{c}\text { Step } 2 \\
\text { Impacts }(\mathbf{p} \text {-value) }\end{array}$} & \multicolumn{3}{|c|}{$\begin{array}{c}\text { Step } 3 \\
\text { Impacts ( } p \text {-value) }\end{array}$} \\
\hline & Direct & Indirect & Total & Direct & Indirect & Total & Direct & Indirect & Total \\
\hline \multicolumn{10}{|l|}{ North } \\
\hline Population (x 1,000, log) & $\begin{array}{c}1.912 \\
(0.394)\end{array}$ & $\begin{array}{c}1.014 \\
(0.413)\end{array}$ & $\begin{array}{c}2.926 \\
(0.396)\end{array}$ & $\begin{array}{c}1.122 \\
(0.584)\end{array}$ & $\begin{array}{c}0.568 \\
(0.635)\end{array}$ & $\begin{array}{c}1.690 \\
(0.599)\end{array}$ & $\begin{array}{l}-0.320 \\
(0.803)\end{array}$ & $\begin{array}{l}-0.100 \\
(0.808)\end{array}$ & $\begin{array}{l}-0.420 \\
(0.803)\end{array}$ \\
\hline GDP per capita (x 1,000 BRL) & $\begin{array}{c}0.153 \\
(0.629)\end{array}$ & $\begin{array}{c}0.081 \\
(0.654)\end{array}$ & $\begin{array}{c}0.234 \\
(0.635)\end{array}$ & $\begin{array}{c}0.065 \\
(0.791)\end{array}$ & $\begin{array}{c}0.033 \\
(0.785)\end{array}$ & $\begin{array}{c}0.097 \\
(0.788)\end{array}$ & $\begin{array}{c}0.052 \\
(0.883)\end{array}$ & $\begin{array}{c}0.016 \\
(0.900)\end{array}$ & $\begin{array}{l}0.068 \\
(0.886)\end{array}$ \\
\hline Gini index (\%) & $\begin{array}{l}-0.063 \\
(0.942)\end{array}$ & $\begin{array}{l}-0.034 \\
(0.982)\end{array}$ & $\begin{array}{l}-0.097 \\
(0.956)\end{array}$ & $\begin{array}{c}0.012 \\
(0.997)\end{array}$ & $\begin{array}{c}0.006 \\
(0.966)\end{array}$ & $\begin{array}{c}0.018 \\
(0.987)\end{array}$ & $\begin{array}{l}-0.124 \\
(0.810)\end{array}$ & $\begin{array}{l}-0.039 \\
(0.835)\end{array}$ & $\begin{array}{l}-0.162 \\
(0.815)\end{array}$ \\
\hline Urbanization rate (\%) & $\begin{array}{c}0.246 \\
(0.075)\end{array}$ & $\begin{array}{c}0.130 \\
(0.108)\end{array}$ & $\begin{array}{c}0.376 \\
(0.080)\end{array}$ & $\begin{array}{c}0.152 \\
(0.383)\end{array}$ & $\begin{array}{c}0.077 \\
(0.411)\end{array}$ & $\begin{array}{c}0.230 \\
(0.390)\end{array}$ & $\begin{array}{c}0.036 \\
(0.742)\end{array}$ & $\begin{array}{c}0.011 \\
(0.756)\end{array}$ & $\begin{array}{r}0.047 \\
(0.743)\end{array}$ \\
\hline FHS coverage (\%) & & & & $\begin{array}{l}-0.021 \\
(0.938)\end{array}$ & $\begin{array}{l}-0.011 \\
(0.903)\end{array}$ & $\begin{array}{l}-0.032 \\
(0.925)\end{array}$ & $\begin{array}{l}-0.069 \\
(0.474)\end{array}$ & $\begin{array}{l}-0.021 \\
(0.526)\end{array}$ & $\begin{array}{l}-0.090 \\
(0.482)\end{array}$ \\
\hline $\begin{array}{l}\text { Radiologists ratio per } 100,000 \\
\text { inhabitants }\end{array}$ & & & & $\begin{array}{c}0.777 \\
(0.641)\end{array}$ & $\begin{array}{c}0.393 \\
(0.648)\end{array}$ & $\begin{array}{c}1.170 \\
(0.641)\end{array}$ & $\begin{array}{c}0.672 \\
(0.671)\end{array}$ & $\begin{array}{c}0.210 \\
(0.665)\end{array}$ & $\begin{array}{r}0.881 \\
(0.667)\end{array}$ \\
\hline $\begin{array}{l}\text { Mammography machines ratio per } \\
10,000 \text { inhabitants }\end{array}$ & & & & $\begin{array}{l}1.663 \\
(0.135)\end{array}$ & $\begin{array}{c}0.842 \\
(0.171)\end{array}$ & $\begin{array}{l}2.505 \\
(0.140)\end{array}$ & $\begin{array}{c}2.089 \\
(0.037)\end{array}$ & $\begin{array}{c}0.652 \\
(0.091)\end{array}$ & $\begin{array}{r}2.741 \\
(0.040)\end{array}$ \\
\hline $\begin{array}{l}\text { Mammograms per mammography } \\
\text { machine }(x 100)\end{array}$ & & & & & & & $\begin{array}{c}1.148 \\
(0.014)\end{array}$ & $\begin{array}{c}0.358 \\
(0.041)\end{array}$ & $\begin{array}{r}1.507 \\
(0.014)\end{array}$ \\
\hline
\end{tabular}

FHS: Family Health Strategy; GDP: Gross Domestic Product.

* The spatial Chow test was significant $(p<0.001)$ for each of the models fitted according to the three steps, indicating evidence for regional dependence in the model's coefficients.

\section{Discussion}

In 2010-2011, mammographic coverage among women aged 50-69 years for the majority of the 438 health micro-regions of Brazil was much lower (median = 21.6\%; IQR: $8.1 \%-37.9 \%$ ) than the 70\%-75\% coverage recommended by the World Health Organization (WHO) 7 and the European guidelines 8 . There were, however, marked inequities concerning coverage ranging from $<1 \%$ to $84 \%$ among health micro-regions of the country, highlighting large geographical inequities in the access to, and uptake of, this public health intervention. Higher coverage was observed in the more socioeconomic developed micro-regions, which were also the ones with smaller wealth inequalities. Area-based associations between socioeconomic variables and mammographic coverage were, as expected, greatly mediated by the more proximal provision of health care and healthcare services utilization variables; but, notably, the association with the Gini index persisted even after adjustment for the latter variables, strongly emphasizing the role of wealth inequalities. The findings also provided strong evidence of spatial correlations, with exposures directly affecting mammographic coverage in a micro-region as well as indirectly through their influence on neighboring micro-regions.

Extensions of the spatial regime model to include region-specific effects showed that correlates of mammographic coverage varied according to the region to which a micro-region belonged. In the South and Southeast, the two most developed regions, household income inequality (as observed by the Gini index), as well as all the provision of health care and healthcare utilization variables, were found to be independent correlates of mammography coverage. In the North and Northeast, the two less developed regions, the only variables found to be independently associated with coverage were the number of radiologists/population and the number of mammograms/machine, indicating that low human resources and poor availability of equipment may be more critical than wealth inequalities.

Our findings are consistent with those from previous studies. In an ecological study using data from a telephone-based survey conducted among adults living in the capitals of the 26 Brazilian states, a positive correlation was observed between the human development index, an area-based marker of socioeconomic development, and self-reported mammographic examination among women aged 
50-69 years (correlation coefficient $[\mathrm{r}]=0.66$ for having had a mammographic examination in the two years prior to the survey) 28 . In individual-based studies, mammographic coverage was found in one city in the Northeast to be higher among better educated women and those with private health insurance 29 and, in another study in the South region, among those living in urban areas 30 .

Data from the 2003 and 2008 Brazilian national household surveys showed that self-reported mammographic screening among women aged 50-69 years increased between surveys (from 54.6\% to 71.5\%), and was positively associated with family income, education, being married, having consulted a doctor, and having private health insurance 31 . Similarly, data from the 2013 national household survey demonstrated that self-reported mammographic screening in the previous two years was higher among women living in the South and Southeast and those of white ethnicity, better educated, and with private health insurance 32 . The low coverage of mammographic screening in Brazil reflects, at least in part, its opportunistic implementation.

Socioeconomic indicators - the most distal variables in the evaluated models - were, with the exception of GDP per capita, associated with mammographic coverage. Coverage was massive in the largest urban centers, which were also the least socially unequal. A study based on data for the 161 IBGE intermediate regions of urban articulation in Brazil, for the years 2008-2015, also showed smaller mammographic screening coverage to be associated with being located in the North and Northeast regions, lower Human Development Index (HDI) and higher Gini coefficient 33. Countries with higher HDI have a higher percentage of breast cancer cases diagnosed in stage I, which is an indicator that they were probably detected by mammographic screening ${ }^{34}$. Authors of a systematic review on the association between area-based socioeconomic indicators and coverage of cancer screening found a great heterogeneity of methods and used indicators and outcomes 35 . For breast cancer, this review found predominantly positive area-based associations between socioeconomic indicators and screening coverage, but with these being attenuated according to adjustment for individual-level covariates, suggesting that the area-based associations may operate, at least in part, through individual-level factors; further studies, with appropriate conceptual models, are needed to clarify this information.

Estimates of mammographic coverage from previous studies tended to be higher than those reported here. There may be several reasons for this difference. First, we aimed to provide coverage estimates for micro-regions, not a national estimate. The latter would require calculation of a weighted mean of the micro-region estimates considering as weights the number of SUS-dependent women aged 50-69 years in each micro-region. Second, we excluded users of private healthcare services, whereas previous research included them. Third, in contrast to our study, which was based on SUS records, previous ones relied on self-reports of past screening experiences, which are likely to be prone to recall errors and even bias. Finally, some studies refer to a more recent period and it is conceivable that coverage might have increased since 2010-2011.

The regression models used in our study considered spatial dependence. The magnitude of indirect impacts of the exposure variables was lower, as expected, than the magnitude of their direct impacts. Nevertheless, modest indirect impacts were observed for most exposures, consistent with interactions between neighboring micro-regions, possibly reflecting transfers of knowledge, behaviors, and people across borders, but also the fact that the socioeconomic level, and the healthcare system, of a micro-region was likely to have influenced its neighboring micro-regions.

Our study has several strengths. The SUS mammographic data we collected were populationbased and thus representative of all women resident in each health micro-region who relied exclusively on SUS, the public health system. SUS data are likely to be complete, since payment to providers depends on them submitting the necessary information. The quality of the exposure data is high, as demonstrated in previous ecological studies 36,37 . Our study has also some limitations. Its ecological design allowed identification of area-level correlations, but not causality. The quality of the SUS mammographic data might have been compromised by several factors. In particular, the SUS database contains information on the number of examinations performed rather than the number of women screened, and hence our estimates of coverage might have been inflated if some women were screened more than once over the 2-year period. A study conducted in the micro-region of Juiz de Fora, in the Southeast region, showed that the time interval between a normal mammogram undertook in 2010 and a subsequent one was $<18$ months for $20 \%$ SUS women ${ }^{38}$. Finally, information on whether mam- 
mography was performed as a screening or diagnostic test was unreliable, and hence all examinations recorded in the SUS database were included in the analyses; however, diagnostic mammograms are likely to have constituted a small fraction of the total mammograms performed in any micro-region except, perhaps, in the few with cancer reference hospitals where a high volume of diagnostic mammograms was performed. We could not directly estimate mammographic coverage and, thus, a proxy variable was used as detailed in the Methods section.

Nevertheless, the study contributes to the emerging literature, showing that women who live in more socioeconomic disadvantaged areas are less likely to access breast cancer screening. Inequities in access to mammographic screening have also been found in high-income countries 10,11,12,13,14,15,39, including in those with free universal health care 10,12,14,15,37. A decline in breast cancer mortality, which began in the 1990s (i.e., prior to the introduction of the screening program) has been observed in the capitals of the South and Southeast regions of Brazil, the most developed in the country 36 , perhaps reflecting better access of symptomatic cases to early detection and treatment (i.e., downward stage migration) in these capitals, and also greater access of asymptomatic women to screening in the private health sector and, more recently, in the public sector as well.

The marked inequities in the access to, and uptake of, mammography throughout Brazil indicate an unequal distribution of barriers limiting access to screening. Tackling these inequalities will be crucial to ensure that mammographic screening will lead to reductions in breast cancer mortality. Further investigations are required to identify and develop locally-appropriate and culturally-sensitive interventions to improve access to, and uptake of, mammographic screening by, for instance, improving screening services and by encouraging women's participation through information and invitation strategies. An important first step towards the development of such appropriate interventions would be the conduct of in-depth studies on barriers to mammographic screening uptake across multiple health micro-regions. Such studies will provide the necessary evidence on woman-level and health system-level barriers to screening uptake, and the extent to which they vary across microregions, and will shed light on why some micro-regions have been able to achieve better screening coverage than others.

\section{Contributors}

M. C. Nogueira contributed to the study design, analysis and interpretation of data, writing the article and final approval of the version to be published. V. A. Fayer, C. S. L. Corrêa, M. R. Guerra, B. De Stavola, I. dos-Santos-Silva, M. T. BustamanteTeixeira and G. Azevedo e Silva contributed to the study design, analysis and interpretation of data, relevant critical review of intellectual content and final approval of the version to be published. All authors declare being responsible for all aspects of the study in ensuring the accuracy and integrity of any part of the article.

\section{Additional informations}

ORCID: Mário Círio Nogueira (0000-0001-96884557); Vívian Assis Fayer (0000-0003-2529-5039); Camila Soares Lima Corrêa (0000-0003-43157123); Maximiliano Ribeiro Guerra (0000-00030234-7190); Bianca De Stavola (0000-0001-78530528); Isabel dos-Santos-Silva (0000-0002-65968798); Maria Teresa Bustamante-Teixeira (00000003-0727-4170); Gulnar Azevedo e Silva (00000001-8734-2799).

\section{Acknowledgments}

This study was conducted as part of a Newtonfunded project on inequalities in women's access to breast and cervical control activities (Research Councils UK and CONFAP Brazil). 


\section{References}

1. Bray F, Ferlay J, Soerjomataram I, Siegel RL, Torre LA, Jemal A. Global cancer statistics 2018: GLOBOCAN estimates of incidence and mortality worldwide for 36 cancers in 185 countries. CA Cancer J Clin 2018; 68:394-424.

2. Shapiro S. Evidence on screening for breast cancer from a randomized trial. Cancer 1977; 39:2777-82.

3. Tabár L, Fagerberg CJ, Gad A, Baldetorp L, Holmberg LH, Grontoft O, et al. Reduction in mortality from breast cancer after mass screening with mammography. Randomised trial from the Breast Cancer Screening Working Group of the Swedish National Board of Health and Welfare. Lancet 1985; 1:829-32.

4. Andersson I, Aspegren K, Janzon L, Landberg T, Lindholm K, Linell F, et al. Mammographic screening and mortality from breast cancer: the Malmö mammographic screening trial. BMJ 1988; 297:943-8.

5. Roberts MM, Alexander FE, Anderson TJ, Chetty U, Donnan PT, Forrest P, et al. Edinburgh trial of screening for breast cancer: mortality at seven years. Lancet 1990; 335:241-6.

6. Miller AB, To T, Baines CJ, Wall C. The Canadian National Breast Screening Study-1: breast cancer mortality after 11 to 16 years of followup. A randomized screening trial of mammography in women age 40 to 49 years. Ann Intern Med 2002; 137(5 part 1):305-12.

7. International Agency for Research on Cancer. Breast cancer screening. IARC handbooks for cancer prevention. v. 7. Lyon: IARC Press; 2002.

8. Perry N, Broeders M, Wolf C, Tornberg S, Holland R, von Karsa L. European guidelines for quality assurance in breast cancer screening and diagnosis. 4th Ed. Luxembourg: Publications Office of the European Union; 2006.

9. Coughlin SS, Leadbetter S, Richards T, Sabatino SA. Contextual analysis of breast and cervical cancer screening and factors associated with health care access among United States women, 2002. Soc Sci Med 2008; 66:260-75.

10. Zidar MN, Larm P, Tillgren P, Akhavan S. Non-attendance of mammographic screening: the roles of age and municipality in a population-based Swedish sample. Int J Equity Health 2015; 14:157.

11. Calo WA, Vernon SW, Lairson DR, Linder SH. Area-level socioeconomic inequalities in the use of mammography screening: a multilevel analysis of the health of Houston Survey. Womens Health Issues 2016; 26:201-7.

12. Azevedo e Silva G, Bustamante-Teixeira MT, Guerra MR, Moura L. Tendências e controle do câncer e os 20 anos de Sistema Único de Saúde no Brasil. In: Ministério da Saúde, organizador. Saúde Brasil 2008: 20 anos de Sistema Único de Saúde (SUS) no Brasil. Brasília: Ministério da Saúde; 2009. p. 365-84.

13. Schootman M, Jeffe DB, Baker EA, Walker MS Effect of area poverty rate on cancer screening across US communities. J Epidemiol Community Health 2006; 60:202-7.
14. Maheswaran R, Pearson T, Jordan H, Black D. Socioeconomic deprivation, travel distance, location of service, and uptake of breast cancer screening in North Derbyshire, UK. J Epidemiol Community Health 2006; 60:208-12.

15. Massat NJ, Douglas E, Waller J, Wardle J, Duffy SW. Variation in cervical and breast cancer screening coverage in England: a cross-sectional analysis to characterize districts with atypical behavior. BMJ Open 2015; 5:e007735

16. Instituto Nacional de Câncer José Alencar Gomes da Silva. Estimativa 2018: incidência de câncer no Brasil. Rio de Janeiro: Instituto Nacional de Câncer José Alencar Gomes da Silva; 2017.

17. Instituto Nacional de Câncer. Controle do câncer de mama - documento de consenso. Rev Bras Cancerol 2004; 50:77-90.

18. Instituto Brasileiro de Geografia e Estatística. Um panorama da saúde no Brasil: acesso e utilização dos serviços, condições de saúde e fatores de risco e proteção à saúde 2008. Rio de Janeiro: Instituto Brasileiro de Geografia e Estatística; 2010.

19. Brasil. Lei no 7.508, de 28 de junho de 2011. Regulamenta a Lei no 8.080, de 19 de setembro de 1990, para dispor sobre a organização do Sistema Único de Saúde - SUS, o planejamento da saúde, a assistência à saúde e a articulação interfederativa, e dá outras providências. Diário Oficial da União 2011; 29 jun.

20. Instituto Nacional de Câncer José Alencar Gomes da Silva. Ficha técnica de indicadores relativos às ações de controle do câncer de mama. Rio de Janeiro: Instituto Nacional de Câncer José Alencar Gomes da Silva; 2014.

21. Instituto Brasileiro de Geografia e Estatística. Divisão regional do Brasil. http://www.ibge. gov.br/home/geociencias/geografia/default_ div_int.shtm?c=1 (accessed on 21/Mar/2017).

22. Almeida E. Econometria espacial aplicada. Campinas: Alínea; 2012.

23. Victora CG, Huttly SR, Fuchs SC, Olinto MT. The role of conceptual frameworks in epidemiological analysis: a hierarchical approach. Int J Epidemiol 1997; 26:224-7.

24. Lee LF. Asymptotic distributions of quasemaximum likelihood estimators for spatial autoregressive models. Econometrics 2004; 72:1899-925.

25. Breusch TS, Pagan AR. The Lagrange multiplier test and its applications to model specification in econometrics. Rev Econ Stud 1980; 47:239-53

26. LeSage J, Pace RK. Introduction to spatial econometrics. Boca Raton: CRC Press; 2009.

27. Anselin L. Spatial regression analysis in R: a workbook. Urbana: University of Illinois, 2007.

28. Sadovsky ADI, Poton WL, Reis-Santos B, Barcelos MRB, Silva ICM. Índice de Desenvolvimento Humano e prevenção secundária de câncer de mama e colo do útero: um estudo ecológico. Cad Saúde Pública 2015; 31:153950 . 
29. Lages RB, Oliveira GP, Simeão Filho VM, Nogueira FM, Teles JBM, Vieira SC. Desigualdades associadas à não realização de mamografia na zona urbana de Teresina-Piauí-Brasil, 2010-2011. Rev Bras Epidemiol 2012; 15:73747.

30. Schneider IJC, Giehl MWC, Boing AF, d'Orsi E. Rastreamento mamográfico do câncer de mama no Sul do Brasil e fatores associados: estudo de base populacional. Cad Saúde Pública 2014; 30:1987-997.

31. Oliveira EX, Pinheiro RS, Melo ECP, Carvalho MS. Condicionantes socioeconômicos e geográficos do acesso à mamografia no Brasil, 2003-2008. Ciênc Saúde Colet 2011; 16:364964.

32. Theme Filha MM, Leal MD, Oliveira EF, Esteves-Pereira AP, Gama SG. Regional and social inequalities in the performance of Pap test and screening mammography and their correlation with lifestyle: Brazilian National Health Survey, 2013. Int J Equity Health 2016; 15:136.

33. Bezerra HS, Melo TFV, Barbosa JV, Feitosa EMLC, Sousa LCM. Avaliação do acesso em mamografias no Brasil e indicadores socioeconômicos: um estudo espacial. Rev Gaúcha Enferm 2018; 39:e20180014.

34. Vieira RAC, Biller G, Uemura G, Ruiz CA, Curado MP. Breast cancer screening in developing countries. Clinics 2017; 72:244-53.
35. Pruitt SL, Shim MJ, Mullen PD, Vernon SW, Amick 3rd BC. Association of area socioeconomic status and breast, cervical and colorectal cancer screening: a systematic review. Cancer Epidemiol Biomarkers Prev 2009; 18:2579-99.

36. Girianelli VR, Gamarra CJ, Azevedo e Silva G. Os grandes contrastes na mortalidade por câncer do colo uterino e de mama no Brasil. Rev Saúde Pública 2014; 48:459-67.

37. Gamarra CJ, Valente JG, Silva GA. Magnitude da mortalidade por câncer do colo do útero na Região Nordeste do Brasil e fatores socioeconômicos. Rev Panam Salud Pública 2010; 28:100-6.

38. Rodrigues TB, De Stavola B, Bustamante-Teixeira MT, Guerra MR, Nogueira MC, Fayer VA, et al. Sobrerrastreio mamográfico: avaliação a partir de bases identificadas do Sistema de Informação do Câncer de Mama (SISMAMA). Cad Saúde Pública 2019; 35:e00049718.

39. Deandrea S, Molina-Barceló A, Uluturk A, Moreno J, Neamtiu L, Peiró-Pérez R, et al. Presence, characteristics and equity of access to breast cancer screening programmes in 27 European countries in 2010 and 2014. Results from an international survey. Prev Med 2016; 91:250-63. 


\section{Resumo}

O estudo teve como objetivos descrever a distribuição espacial do rastreamento por mamografia entre áreas geográficas pequenas (microrregiões) no Brasil, além de investigar se as diferenças observadas estavam associadas a inequidades espaciais nas condições socioeconômicas, na prestação de assistência à saúde e no uso de serviços de saúde. Este foi um estudo ecológico de base territorial, comparando a cobertura do rastreamento por mamografia em 2010-2011 com fatores socioeconômicos e de cuidados de saúde. O estudo usou 438 microrregiões sanitárias brasileiras como as unidades analíticas. Foram utilizados modelos de regressão espacial para estudar as associações. Houve uma importante variabilidade na cobertura por mamografia entre microrregiões (mediana $=$ 21,6\%; variação interquartil: 8,1\%-37,9\%). A análise multivariada identificou: forte desigualdade na renda familiar, número baixo de radiologistas/100 mil habitantes, número baixo de aparelhos de mamografia/10 mil habitantes e número baixo de mamografias realizadas com cada aparelho enquanto correlatos independentes da baixa cobertura mamográfica no nível microrregional. Houve evidência de forte dependência espacial nessas associações, em que as mudanças em uma microrregião afetavam as microrregiões vizinhas, além de heterogeneidade geográfica. O estudo revelou importantes inequidades no acesso ao exame de mamografia entre microrregiões brasileiras em 2010-2011, com cobertura mais alta nas microrregiões com menor desigualdade de renda e melhor acesso geral aos cuidados de saúde.

Iniquidade em Saúde; Neoplasias da Mama; Acesso aos Serviços de Saúde

\section{Resumen}

Los objetivos de este estudio fueron describir la distribución espacial de la cobertura del cribado mamográfico, a través de pequeñas áreas geográficas (microrregiones) en Brasil, y examinar si las diferencias observadas estuvieron asociadas con inequidades espaciales, en términos de condiciones socioeconómicas, sistema de atención de salud, y utilización de servicios de salud. Se trata de un estudio ecológico, basado en áreas incluidas en la cobertura de cribado mamográfico durante 20102011 y relacionadas con variables socioeconómicas $y$ de salud. Las unidades de análisis fueron 438 microrregiones de salud en Brasil. Se utilizaron modelos de regresión espacial para estudiar estas relaciones existentes. Hubo una variabilidad marcada en relación con la cobertura mamográfica a través de las microrregiones (media $=21.6 \%$; rango intercuartílico: 8,1\%-37,9\%). Los análisis multivariables identificaron una alta inequidad en los ingresos por hogar, bajo número de radiólogos/100,000 habitantes, bajo número de máquinas de mamografia/10.000 habitantes, y un bajo número de mamografías realizadas por cada máquina, lo que está independiente correlacionado con la baja cobertura de mamografías en el nivel de microrregión. Hubo evidencias de una dependencia espacial fuerte de estas asociaciones, con cambios en una microrregión afectando a microrregiones vecinas, y también de heterogeneidades geográficas. Hubo inequidades sustanciales en el acceso al cribado mamográfico a través de las microrregiones en Brasil, en 2010-2011, con una cobertura superior en aquellas con pequeñas inequidades respecto a la riqueza y mejor acceso a los servicios de salud.

Inequidad en Salud; Neoplasias de la Mama; Accesibilidad a los Servicios de Salud
Submitted on 10/Jun/2017

Final version resubmitted on 29/Jan/2019

Approved on 06/Feb/2019 